\title{
Effect of polyethylene glycol and propyltrimethoxysilane on structural and optical properties of zinc oxide nanoparticles synthesized by sol- gel process
}

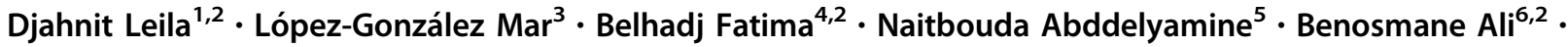 \\ Haddaoui Nacereddine ${ }^{1}$
}

Received: 15 January 2018/Accepted: 14 September 2018/Published online: 5 October 2018

(c) The Author(s) 2018

\begin{abstract}
Zinc oxide nanoparticles (ZnO NPs) are prepared by sol-gel process, using both polyethylene glycol (PEG-400) as surfactant and propyltrimethoxysilane (PTMS) as capping agent. Surface modification is performed in situ procedure. The physical parameters such as strain and stress values are calculated via the Williamson-Hall plot $(\mathrm{W} \& \mathrm{H})$ assuming a uniform deformation model (UDM) and uniform stress deformation model, and by the size and strain plot method (SSP). The results show that the crystallite size estimated from Scherrer's formula, (W\&H), (UDM), (SSP) and the particle size estimated from DSL are inter-correlated, which confirm the small size and the isotropic nature of our ZnO NPs. The FTIR spectroscopy illustrates that PEG-400 and PTMS could be adsorbed at the ZnO NPs surface. The distinct emission peak in the blue band is located at $490 \mathrm{~nm}$ and $E_{2}$ (high) mode is situated at $436 \mathrm{~cm}^{-1}$. Both results confirm the oxygen deficiency in the $\mathrm{ZnO}$ NPs.
\end{abstract}

Keywords Sol-gel synthesis · Zinc oxide · PEG-400 · Propyltrimethoxysilane · Nanoparticles · DRX

\section{Introduction}

Zinc oxide $(\mathrm{ZnO})$ is known as an important semiconductor for its favorable properties as transparency [1], high electron mobility [2], wide band gap [3], tough room

Djahnit Leila

leila_djahnit@yahoo.fr

1 Laboratoire de Physico-Chimie des Hauts Polymères, Université de Ferhat Abbes, Sétif-1, Sétif, Algeria

2 Département de Chimie, Université de Hassiba Benbouali, Chlef, Algeria

3 Instituto de Ciencia y Tecnología de Polímeros (ICTP-CSIC), Juan de la Cierva, 3, 28006 Madrid, Spain

4 Chemical Materials Laboratory, University Ahmed Benballa, Oran, Algeria

5 Centre de Développement des Technologies Avancées, Algiers, Algeria

6 Laboratory of Molecular Chemistry, Environmental Control and Physico-Chemical Measurements, University of Mentouri, Constantine, Algeria temperature [4] and luminescence [5]. In the last few years, there has been a growing interest of the researchers, focusing on the synthesis of nanocrystalline $\mathrm{ZnO}$, hence, many methods, including sol-gel [6-9]. Thereby, particle size and crystal morphology play important roles in all applications, like nanodevices for opto-electronic [10], gas sensing [11], photocatalytic activity [12]. A part of the technological meaning of zinc oxide nanoparticles $(\mathrm{ZnO}$ $\mathrm{NPs}$ ), their diameters ranging from 10 to $100 \mathrm{~nm}$ make them good-looking from academic point of view [13].

The current paper presents a novel method to use both polyethylene glycol (PEG-400) and propyltrimethoxysilane (PTMS) as surfactant and capping agent, respectively. Because several publications have demonstrated that PEG400 is easily adsorbed at the surface of a metal oxide and modifies the kinetics of the growing colloids. In addition, it is well known that PEG-400 dissolved in alcohol increases the viscosity. This process not only significantly shortens the reaction time for preparing $\mathrm{ZnO}$, but also increases the crystallinity of the ZnO NPs. When PEG-400 is added before synthesis, the probability of electrostatic adsorption of $\mathrm{ZnO}$ NPs increased. It has been proposed to add silane to 
enhance the stability and to prevent $\mathrm{ZnO}$ agglomeration. In this case, it would be important that PTMS could protect the surface against agglomeration in the presence of PEG400 , and control their size and photoemission properties [13-17]. A perfect crystal would extend infinitely in all directions, for that reason, no crystal is perfect due to its finite size. This deviation from perfect crystallinity leads to a broadening of the diffraction peaks. In our study, we explore the possibility to have good crystallinity and small size of $\mathrm{ZnO}$ NPs.

\section{Experimental procedure}

\section{Synthesis of $\mathrm{ZnO}$ nanoparticles}

In order to synthesize $\mathrm{ZnO} \mathrm{NPs}$, the major step is the preparation of the organometallic precursor containing $\left(0.1 \mathrm{M}\right.$ de $\left.\mathrm{Zn}^{2+}\right) \quad\left(\mathrm{C}_{4} \mathrm{H}_{6} \mathrm{O}_{4} \mathrm{Zn} \cdot 2 \mathrm{H}_{2} \mathrm{O}\right.$ from Aldrich) in absolute ethanol, where the solution is refluxed at $80{ }^{\circ} \mathrm{C}$, under continuous stirring and atmospheric pressure. After $2 \mathrm{~h}$, the as-obtained solution is cooled and is stirred about 30 min to ensure good dispersion while adding the PEG400. Consequently, the PTMS is added drop wise to the solution during $40 \mathrm{~min}$. An aliquot of lithium oxide $\left(\mathrm{LiOH} \cdot \mathrm{H}_{2} \mathrm{O}\right)$ solution prepared in ethanol $(0.25 \mathrm{M})$ is dropped to the previous solution. So, a transparent solution containing $\mathrm{ZnO}$ NPs quantum dot is obtained. The resulting white powder is obtained by precipitation of previous solution with hexane. The product is washed by ethanol and bi-distilled water three times and then is centrifuged. At the end, the final solid is dried in an oven.

\section{Characterization}

The crystal structure of $\mathrm{ZnO}$ NPs is analyzed by a powder $\mathrm{X}$-ray diffractometer (XRD) advanced Bruker D8 equipped with a source of X-ray. The UV-Vis absorption spectra are recorded using (Shimadzu UV 2450) UV-Vis. spectrophotometer. The photoluminescence (PL) spectra are recorded at room temperature using Fluorescence Spectrophotometer LS 50B, Perkin Elmer. Fourier transform infrared (FTIR) spectra are recorded using Perkin Elmer. The particle size distribution of the powder is measured by photon correlation spectroscopy (PCS), using a Malvern Zetasizer Nano ZS laser particle size analyzer. Raman spectroscopy is studied by a confocal micro-Raman spectrometer 'LabRam HR 800' by Horiba Jobin-Yvon, in association with an optical Olympus BX41 microscope.

\section{Results and discussion}

\section{X-ray diffraction analysis}

All the peaks in the diffractogram have been indexed according to the Hexagonal wurtzite structure of $\mathrm{ZnO}$. As observed in Fig. 1, the main significant peaks are $31.81^{\circ}$, $34.25^{\circ}, 36.10^{\circ}, 47.38^{\circ}, 56.46^{\circ}, 63.03^{\circ}, 66.64^{\circ}, 67.07^{\circ}$, $68.91^{\circ}, 72.61^{\circ}$ and $76.98^{\circ}$. The peaks are indexed as (100), (002), (101), (102), (110), (103), (200), (112), (201), (004) and (202), respectively. These results are consistent with the Committee of Powder Diffraction Standards, JCPDS No. (36-1451). The observed diffractogram shows high and wide peaks, indicating high crystallinity and small size particles. The lattice parameter $a$ and the ratio of the lattice parameters $c / a$ are constant for a given diffraction pattern, so $\frac{\lambda^{2}}{4 a^{2}}$ is constant for a Hexagonal structural pattern, that can be indexed considering $\frac{4}{3}\left(h^{2}+h k+k^{2}\right)$ and $\frac{l^{2}}{\frac{c^{2}}{a^{2}}}$ terms in the next equation

$\sin ^{2} \theta=\frac{\lambda^{2}}{4 a^{2}} \frac{4}{3}\left(h^{2}+h k+k^{2}\right)+\frac{l^{2}}{\frac{c^{2}}{a^{2}}}$

The summations of the two terms for permissible $(h k l)$ planes are arranged in their ascending order as presented in Table 1 . We estimate the lattice parameters $a=b$ and $c$, and crystallographic axes with angles $\alpha=\beta=90^{\circ}$, $\gamma=120^{\circ}$. Here, $a$ and $c$ are calculated as following $[18,19]$

$a=\frac{\lambda}{\sqrt{3}} \frac{1}{\sin \theta}\left(\sqrt{\left(h^{2}+h k+k^{2}\right.}\right)$

$c=\frac{\lambda}{2 \sin \theta} l$

The results are summarized in Table 2, where the average values of the calculated lattice constants are

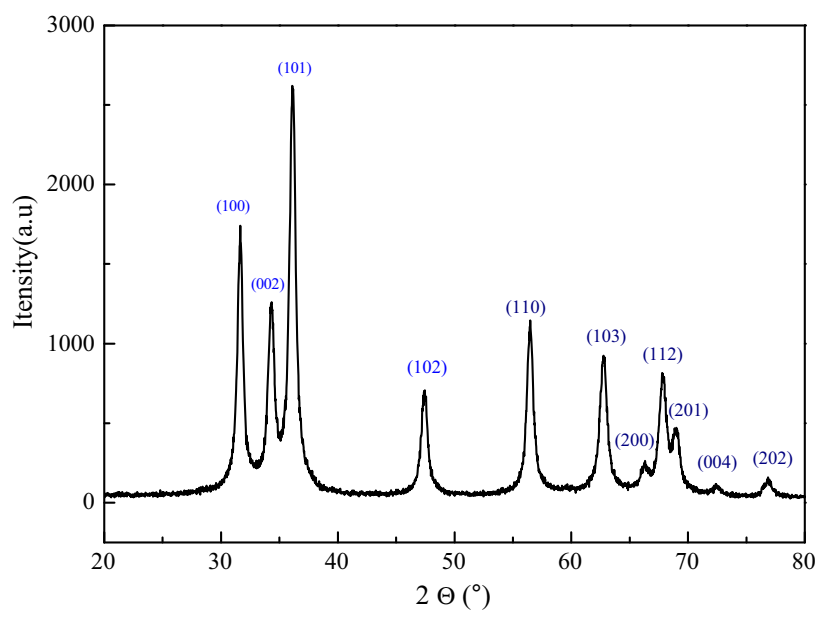

Fig. 1 The X-ray diffraction pattern of $\mathrm{ZnO}$ nanoparticles 
Table 1 Miller indices $(h k l)$ planes for $\mathrm{ZnO} \mathrm{NPs}$

\begin{tabular}{llll}
\hline$h$ & $k$ & $l$ & $\frac{4}{3}\left(h^{2}+h k+k^{2}\right)+\frac{l^{2}}{\frac{c^{2}}{a^{2}}}$ \\
\hline 1 & 0 & 0 & 1.333 \\
0 & 0 & 2 & 1.5489 \\
1 & 0 & 1 & 1.7205 \\
1 & 0 & 2 & 2.8819 \\
1 & 1 & 0 & 4 \\
1 & 0 & 3 & 4.8184 \\
2 & 0 & 0 & 5.333 \\
1 & 1 & 2 & 5.5489 \\
2 & 1 & 1 & 5.7205 \\
0 & 0 & 4 & 6.1958 \\
2 & 0 & 2 & 6.8822 \\
\hline
\end{tabular}

Table 2 Values of lattice parameters

\begin{tabular}{llllllll}
\hline $2 \theta$ & $\theta$ & $d(\AA)$ & $h k l$ & $h^{2}+h k+k^{2}$ & $l^{2}$ & $a(\AA)$ & $c(\AA)$ \\
\hline 31.81 & 15.905 & 2.8108 & 100 & 1 & 0 & 3.2457 & \\
34.25 & 17.12 & 2.616 & 002 & 0 & 4 & & 5.2322 \\
36.10 & 18.05 & 2.4865 & 101 & 1 & 1 & & \\
47.38 & 23.69 & 1.9172 & 102 & 1 & 4 & & \\
56.46 & 28.23 & 1.6285 & 110 & 3 & 0 & 3.2571 & \\
63.03 & 31.51 & 1.474 & 103 & 1 & 9 & & \\
66.64 & 33.32 & 1.4023 & 200 & 4 & 0 & 3.2385 & \\
68.07 & 34.034 & 1.3762 & 112 & 3 & 4 & & \\
68.91 & 34.45 & 1.3616 & 201 & 4 & 1 & & \\
72.61 & 36.305 & 1.3001 & 004 & 0 & 16 & & 5.2039 \\
76.98 & 38.49 & 1.2376 & 202 & 4 & 4 & & \\
\hline
\end{tabular}

$a=3.24709 \AA$ and $c=5.21807 \AA$. The average size of the $\mathrm{ZnO}$ nanocrystals is determined using the Debye-Scherrer formula $[20,21]$

$\tau=\frac{k \lambda}{\beta \cos \theta_{\beta}}$

where $\tau$ presents the particle size diameter in nanometer. $k$ is a constant depending on the shape of the particles and is equal to 0.89. $\lambda, \beta$ and $\theta_{\beta}$ are the wavelength of $C u k_{\alpha}$ radiation, the full width at half maxima (FWHM) and the Bragg diffraction angle in radian, respectively. The data of crystal size are illustrated in Table 3 . The nanocrystals $\mathrm{ZnO}$ size of the sample are found to be $10.6 \mathrm{~nm}$, which is derived from the FWHM of the most intense peak corresponding to (101) plane located at $36.094^{\circ}$. In addition, other wurtzite lattice parameters such as the interplanar angle and the unit cell volume could also be considered through Lattice Geometry [21], given by Eqs. 5 and 6 as follows:
Table 3 The crystal size using the Debye-Scherrer relation

\begin{tabular}{lllr}
\hline $2 \theta$ & $h k l$ & $\beta(\mathrm{rad})$ & $\tau(\mathrm{nm})$ \\
\hline 31.81 & 100 & 0.0165 & 8.3 \\
34.25 & 002 & 0.0204 & 5.6 \\
36.10 & 101 & 0.0129 & 10.6 \\
47.38 & 102 & 0.0126 & 10.8 \\
56.46 & 110 & 0.0131 & 10.5 \\
63.03 & 103 & 0.0215 & 6.4 \\
66.64 & 200 & 0.0441 & 3.1 \\
68.07 & 112 & 0.0191 & 7.2 \\
68.91 & 201 & 0.0093 & 14.7 \\
72.61 & 004 & 0.00005 & 2.7 \\
76.98 & 202 & 0.0088 & 15.5 \\
\hline
\end{tabular}

$\operatorname{Cos} \varphi=\frac{\left(h_{1} h_{2}+k_{1} k_{2}+\frac{1}{2}\left(h_{1} k_{2}+h_{2} k_{1}\right)+\frac{3 a^{2}}{4 c^{2}} l_{1} l_{2}\right)}{\sqrt{\left(h_{1}^{2}+k_{1}^{2}+h_{1} k_{1}+\frac{3 a^{2}}{4 c^{2}} l_{1}^{2}\right)\left(h_{2}^{2}+k_{2}^{2}+h_{2} k_{2}+\frac{3 a^{2}}{4 c^{2}} 2_{2}^{2}\right)}}$

$V=\frac{\sqrt{3}}{2} a^{2} c=0.866 a^{2} c$

Using Eq. 6 [22], the unit cell volume for the prepared $\mathrm{ZnO}$ nanocrystals is estimated to be $47.65 \mathrm{~nm}^{3}$. In addition, from Eq. 5, the interplanar angle between (112) and (201) planes $\varphi$ is found to be $32^{\circ}$ [23].

\section{Size and strain plot}

A better evaluation of the size and strain parameters can be obtained by considering an average size and strain plot (SSP) [23]. In this approximation, it is assumed that the crystallite size and the strain profiles are described by a Lorentzian and a Gaussian functions, respectively [24]. Moreover, the peak broadening due to the lattice strain is estimated from the following equation [25]

$\left(\beta_{h k l} d_{h k l} \cos \theta\right)^{2}=\frac{k}{D}\left(d_{h k l}^{2} \beta_{h k l} \cos \theta\right)+\left(\frac{\varepsilon}{2}\right)^{2}$

where $k$ is a constant that depends on the shape of the nanoparticles and $D$ is the crystalline size. The term $\left(\beta_{h k l} d_{h k l} \cos \theta\right)^{2}$ is plotted versus $\left(d_{h k l}^{2} \beta_{h k l} \cos \theta\right)$ in Fig. 2 . The crystalline size is evaluated from the slope of the linearly fitted data and $y$-intercept gives the strain $\varepsilon$.

\section{Uniform deformation model}

The strain-induced broadening in the nanoparticles due to the crystal imperfection and distortion is calculated using the formula [25]

$\varepsilon=\frac{\beta_{h k l}}{4 \tan \theta}$ 


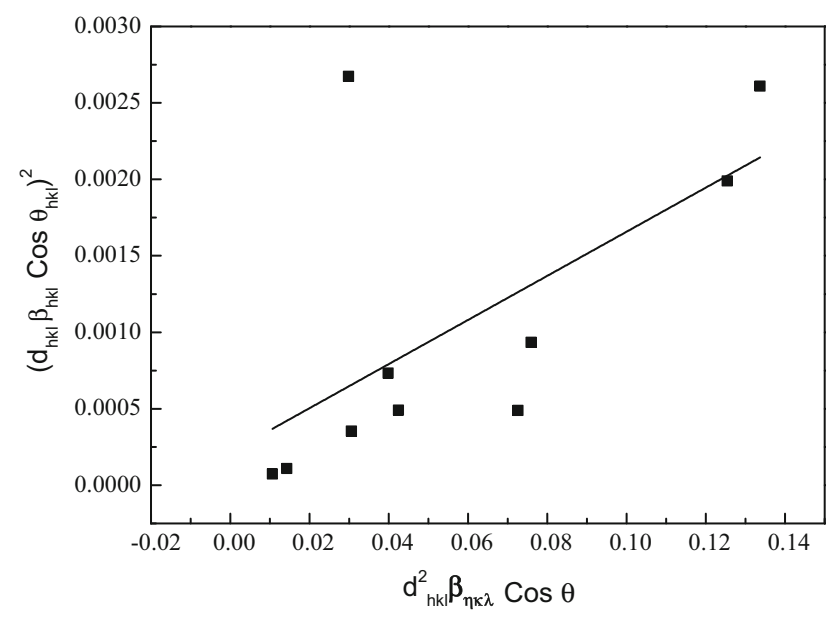

Fig. 2 Average size and strain plot (SSP)

Furthermore, the total broadening is obtained from [26]

$\beta_{h k l}=\beta_{\mathrm{s}}+\beta_{D}$

where $\beta_{D}$ is the peak broadening corresponding to crystallite size, $\beta_{\mathrm{s}}$ is due to strain-induced broadening. $\beta_{h k l}$ is the width of the half-maximum intensity of instrumental corrected broadening. In addition, $\mathrm{W} \& \mathrm{H}$ analysis are simplified methods, which clearly differentiate between size-induced and strain-induced peak broadening by considering the peak width as a function of $2 \theta$. The instrumental corrected broadening of each diffraction peak is calculated using Eq. 9. From the equation of DebyeScherrer, it is confirmed that the peak width of the crystallite size varies as $1 / \cos \theta$, while the strain varies as $\tan \theta$ in the W\&H method [27]

$\beta_{h k l}=\frac{k \lambda}{D \beta_{h k l}}+4 \varepsilon \tan \theta$

By rearranging the above equation, we find

$\beta_{h k l} \cos \theta=\frac{k \lambda}{D}+4 \varepsilon \sin \theta$

The plot of $\beta_{h k l} \cos \theta$ versus $4 \sin \theta$ for as-prepared $\mathrm{ZnO}$ NPs is shown in Fig. 3. From the linear fit, the crystallite size is calculated from the y-intercept and the strain $\varepsilon$ is estimated from the slope of the line plotted. The strain takes negative value or compressive strain, which is most likely due to the lattice shrinkage and we suppose that is related to quantum confinement.

\section{Uniform stress deformation model}

Uniform deformation stress is taken into the anisotropic nature of Young's modulus of the crystal. The generalized Hook's law refers to the strain. By assuming a small strain in $\mathrm{ZnO}$ crystals, so Hooke's law can be applied here. The

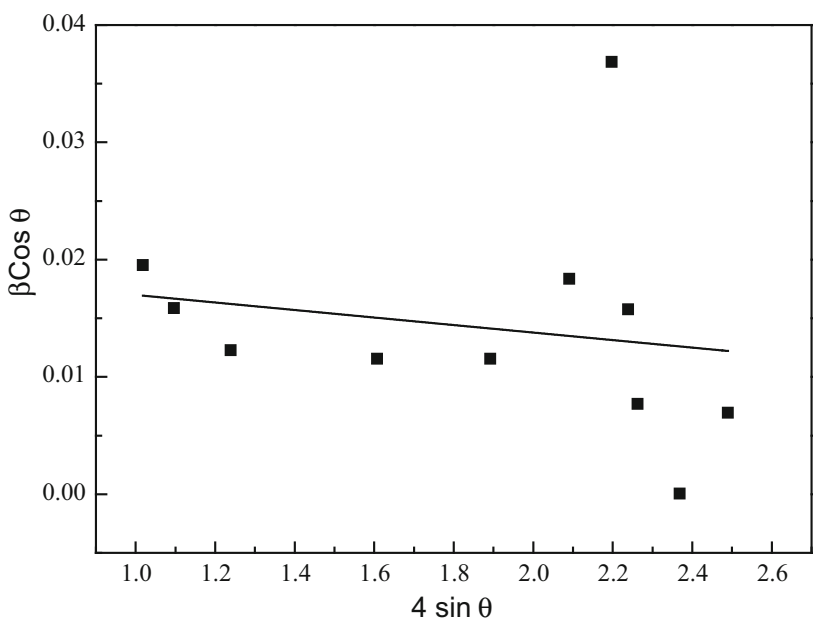

Fig. 3 The $\mathrm{W} \& \mathrm{H}$ analysis of $\mathrm{ZnO}$ NPs using UDM

value of $\sigma$ is determined from the slope of the graph plotted between $\beta_{h k l} \cos \theta$ versus $\frac{4 \sin \theta}{Y_{h k l}}$, represented in Eq. 12. In the USDM, the W\&H equation is simply modified by replacing the value of $\varepsilon$ in the second term of Eq. 12, where

$\beta_{h k l} \cos \theta=\frac{k \lambda}{D}+\frac{4 \sigma \sin \theta}{Y_{h k l}}$

By the linear fit of the data, the crystallite size and the strain are determined from the slope and the intercept, respectively, in Fig. 4. All the results obtained from the Scherrer method, W\&H models (UDM, USDM) and SSP analyses are summarized in Table 4. Hence, while considering the crystallite size, the strain must be taken into account as in the W\&H methods. But when the strain takes negative values the size decreases. By virtue on Hook's law, a linear proportionality between the stress and strain is given as $\sigma=Y \varepsilon$, where $\sigma$ is the stress of the crystal and $Y$ is the Young's modulus. For a Hexagonal crystal, Young's modulus is given by the following relation

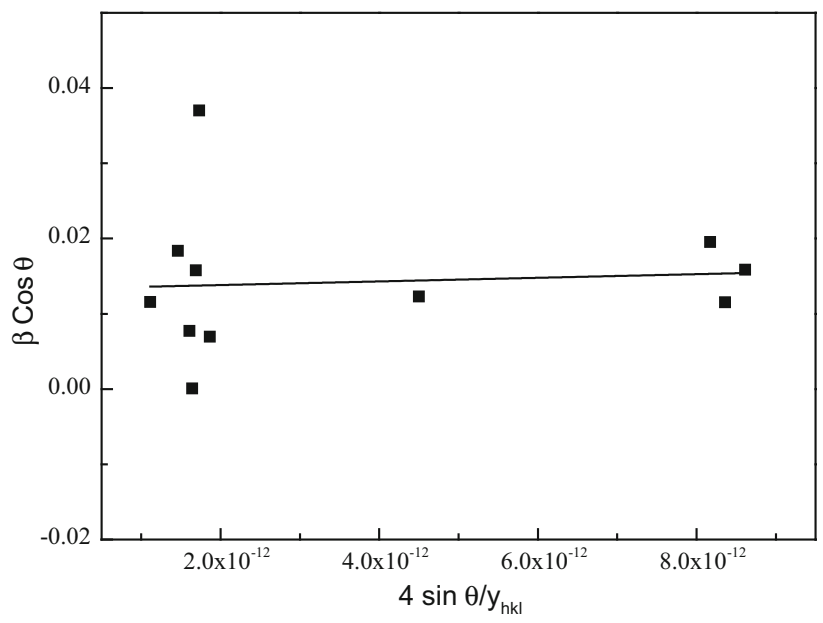

Fig. 4 The W\&H analysis of ZnO NPs using USDM 
$Y_{h k l}=\frac{\left(h^{2}+\frac{(h+2 k)^{2}}{3}+\left(\frac{a l}{c}\right)^{2}\right)^{2}}{S_{11}\left(h^{2}+\frac{(h+2 k)^{2}}{3}\right)^{2}+S_{33}\left(\frac{a l}{c}\right)^{4}+\left(2 S_{13}+S_{44}\right)\left(h^{2}+\frac{(h+2 k)^{2}}{3}+\left(\frac{a l}{c}\right)^{2}\right)^{2}}$

Young's modulus $Y_{h k l}$ is related to their elastic compliances $S_{i j}[23,27]$ where $S_{i j}=S_{11}, S_{13}, S_{33}, S_{44}$ are the elastic compliances of $\mathrm{ZnO}$, with the values of $7.858 \times 10^{-12}, \quad-2.206 \times 10^{-12}, \quad 6.940 \times 10^{-12}$, $23.57 \times 10^{-12} \mathrm{~m}^{2} \mathrm{~N}^{-1}$, respectively. The estimated Young's modulus of $\mathrm{ZnO}$ NPs is equal to $142 \mathrm{GPa}$, which is significantly larger than the bulk value [27-31]. Chen et al. [32] have reported an experimental measurement of the size-dependent of $\mathrm{ZnO}$ nanowires. They found that when the diameter of the nanoparticles is smaller than $120 \mathrm{~nm}$, Young's modulus increases significantly with decreasing diameter. Our result agrees with the experimental trend (Table 4).

\section{Dislocation density and $\mathrm{Zn}-0$ bond length}

A dislocation is a crystallographic defect or irregularity within a crystal structure. The dislocation density symbolized by $\delta$ represents the amount of defects in the powder [21], which is determined from the following equation

$\delta=\frac{1}{\tau^{2}}$

where $\tau$ is the particle size, in our case is found to be equal to $8.68 \mathrm{~nm}$, calculated from Debye-Scherrer method. The $\mathrm{Zn}-\mathrm{O}$ bond length $l$ is given by

$l=\sqrt{\frac{a^{2}}{3}+\left(\frac{1}{2}-u\right)^{2} c^{2}}$

where $u$ is given by

$u={\frac{a}{3 c^{2}}}^{2}+0.25$

Table 4 Summary of all models results

\begin{tabular}{lccc}
\hline & Size $(\mathrm{nm})$ & $\varepsilon \times 10^{-3}$ & $\delta(\mathrm{MPa})$ \\
\hline Debye-Scherrer & 8.6 & & \\
SSP & 5.2 & 7.34 & \\
W\&H & & & \\
UDM & 6.8 & -3.2 & 241 \\
USDM & 10.3 & 17 & \\
\hline
\end{tabular}

The estimated bond length of Zn-O NPs is $1.978 \AA$, and the dislocation density is about $1.32 \times 10^{16} \mathrm{~m}^{-2}$, while the reported bond length is in the range of 1.87-2.01 $\AA$, which reveals the quantum size of the NPs. The structure seems to be a Hexagonal 3D $\mathrm{ZnO}$ chains to form spherical nanoparticles.

\section{Laser light scattering size analyzer}

The Malvern Zetasizer is used to measure the particle size in the colloidal solution of $\mathrm{ZnO}$ NPs dispersed in ethanol. Even that the $\mathrm{ZnO}$ NPs are stored in ambient environment for 14 days, the size distribution remains in narrow range as shown in Fig. 5. The comparison of the results obtained from XRD and DSL methods shows smaller average particle size of about $6 \mathrm{~nm}$, confirming the quantum dot properties.

\section{Fourier Transform infrared spectroscopy}

The spectrum in Fig. 6 shows a broad band at $3476 \mathrm{~cm}^{-1}$, assigned to the $\mathrm{O}-\mathrm{H}$ stretching mode. It is shifted toward low wavenumber that demonstrates the formation of hydrogen bond between the PEG particles and ZnO NPs on their surfaces $[33,34]$. The peak at $1100 \mathrm{~cm}^{-1}$ is due to $\mathrm{C}-\mathrm{O}(\mathrm{H})$ vibration that could qualify the coating PEG [35]. The small bands at 2838 and $2900 \mathrm{~cm}^{-1}$ are due to $\mathrm{C}-\mathrm{H}$ stretching vibration of the alkane groups of PTMS and PEG $[33,36]$. As it can be seen, the peaks centered at 840 and $890 \mathrm{~cm}^{-1}$ are appropriate to $\mathrm{Zn}-\mathrm{O}-\mathrm{Si}$ bonds and $\mathrm{Si}-$ $\mathrm{O}-\mathrm{Si}$ bridges that are formed by in situ self-condensation of the hydrolyzed organosilane molecules [36]. Furthermore, a broad absorption peak at $1000 \mathrm{~cm}^{-1}$ is assigned to $\mathrm{Si}-\mathrm{O}$ vibration as obtained by M. K. Patra et al. [35]. The peak in the region extending from 1250 to $1450 \mathrm{~cm}^{-1}$ is likely due to the bending modes of $\mathrm{O}-\mathrm{H}$. In addition, the characteristic band for $\mathrm{Zn}-\mathrm{O}$ stretching vibrations is shifted to a lower wavenumber of $447 \mathrm{~cm}^{-1}$, that is may be due to the presence of silica $-\mathrm{Si}-\mathrm{O}-\mathrm{Si}-$ network on the $\mathrm{ZnO}$ NPs [37]. There are two peaks at approximately 1587 and $1413 \mathrm{~cm}^{-1}$ correspond to the asymmetric and symmetric stretching vibrations of $\mathrm{C}=\mathrm{O}$ and $\mathrm{C}-\mathrm{O}$ in the acetate $(\mathrm{COO})$ groups, respectively [38]. 


\section{Ultraviolet-visible studies}

Figure 7 depicts the absorption spectrum of $\mathrm{ZnO}$ in fresh ethanolic colloids. The UV-Vis. spectrum shows a band between 250 and $340 \mathrm{~nm}$, with two maxima at $300 \mathrm{~nm}$ and $273 \mathrm{~nm}$, attributed to exciton transitions in small $\mathrm{ZnO}$ clusters. As stated in previous reports [39-41], five pronounced absorption features are typically detected, situated near 210, 230, 270, 320 and $360 \mathrm{~nm}$. These electronic transitions reflect the appearance of preferentially stable "magic sizes" from the II-VI-semiconductor cluster chemistry. Furthermore, Searson et al. [42] have analyzed the progressive spectral between 300 and $360 \mathrm{~nm}$ which is associated with nanoparticle size range between 2 and $6 \mathrm{~nm}$.

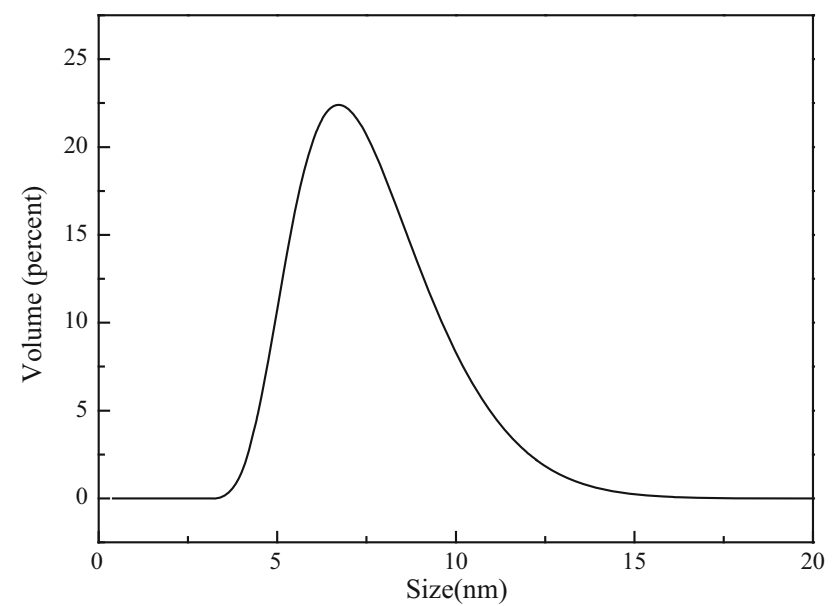

Fig. 5 Particle size distribution of prepared ZnO NPs

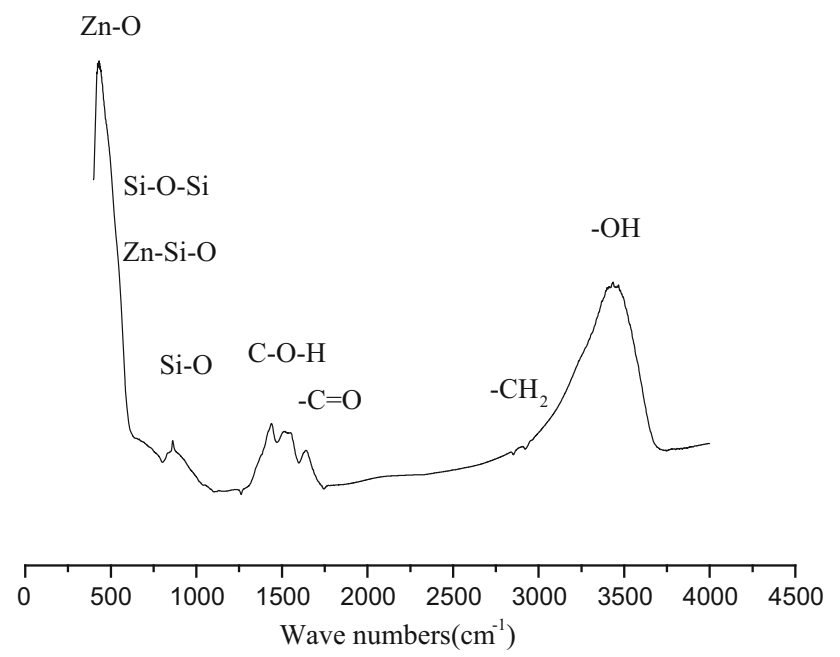

Fig. 6 FTIR full range spectrum of ZnO NPs synthesized

\section{Photoluminescence}

In pure $\mathrm{ZnO}$ NPs spectrum, the emission broad peak from 400 to $620 \mathrm{~nm}$ (blue emission) has been observed in Fig. 8, that is appropriate to surface defect in $\mathrm{ZnO}[43,44]$. It is inferred that the silane surface modification during the growth process could greatly reduce the aggregation and thus increase the effective surface luminescence [45]. It is recognized that the distinct emission peak in the blue band within $490 \mathrm{~nm}$ that is appropriate to the direct recombination of conduction electron in the conduction band and a hole, which may occur from the transition due to oxygen anti-site vacancy defect state [46].

\section{Raman spectroscopy}

To further investigate the optical properties, in Fig. 9, we present a typical non-resonant Raman scattering spectrum

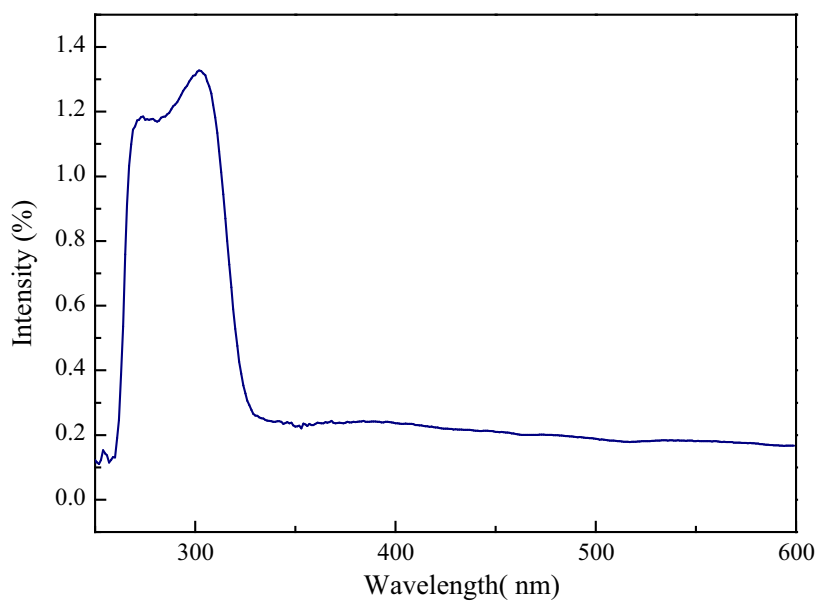

Fig. 7 Optical density of solution colloidal of ZnO NPs synthesized

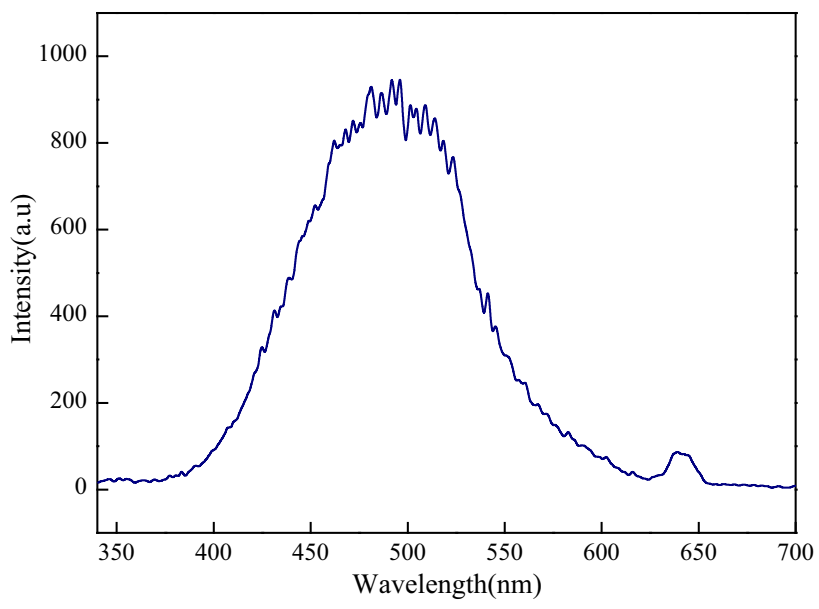

Fig. 8 Luminescence properties of $\mathrm{ZnO}$ nanoparticles 


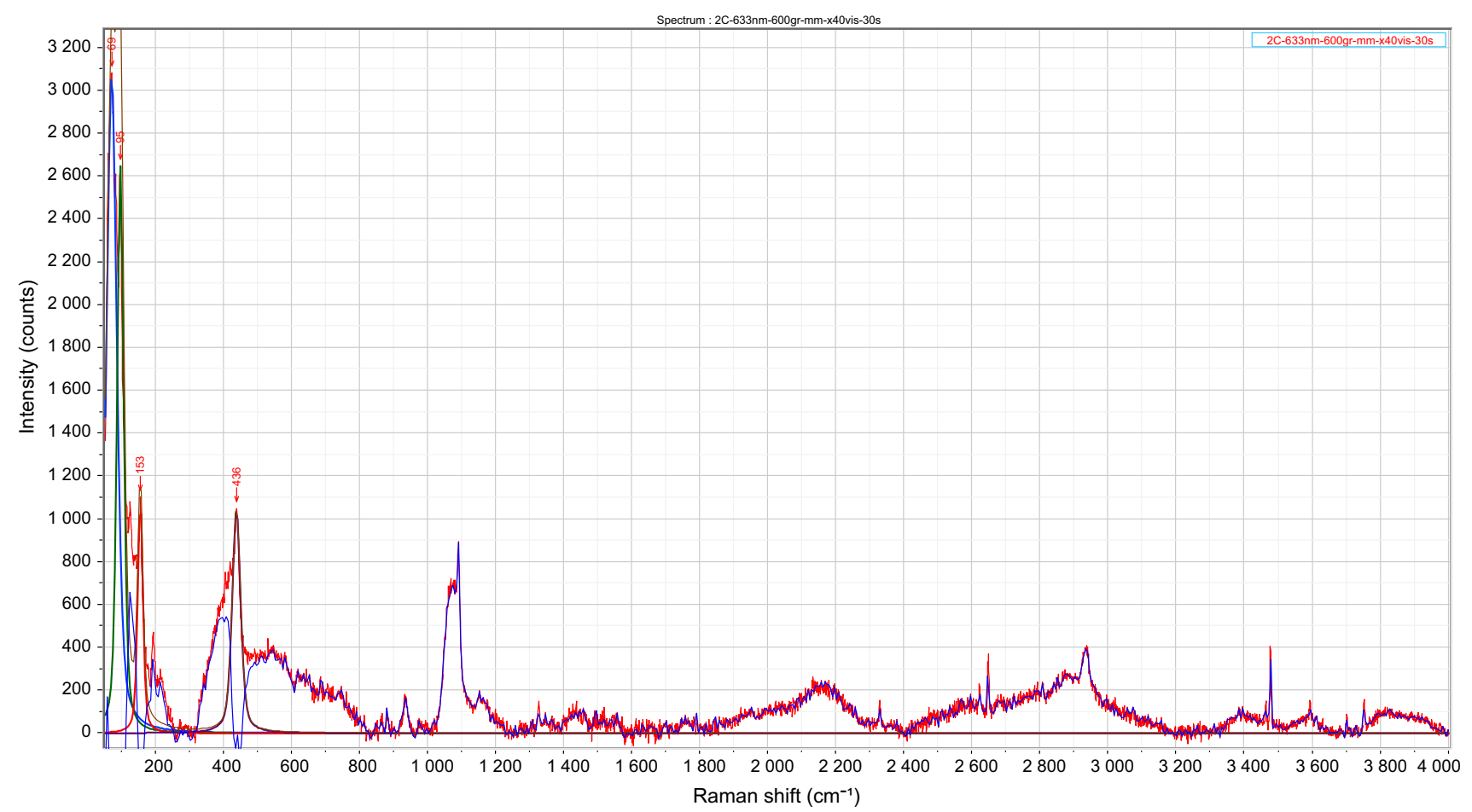

Fig. 9 Raman spectra excited by $633 \mathrm{~nm}$ line of as-grown $\mathrm{ZnO}$ at room temperature (the blue line is the fitting curve)

Table 5 Raman active phonon mode frequencies $\left(\right.$ in $\mathrm{cm}^{-1}$ ) for bulk $\mathrm{ZnO}$ and $\mathrm{ZnO}$ nanoparticles synthesized

\begin{tabular}{lllllll}
\hline & $E_{2}(\mathrm{low})$ & $A_{1}(\mathrm{TO})$ & $E_{1}(\mathrm{TO})$ & $E_{2}(\mathrm{high})$ & $A_{1}(\mathrm{LO})$ & $E_{1}(\mathrm{LO})$ \\
\hline ZnO bulk $^{\mathrm{a}}$ & 102 & 379 & 410 & 439 & 574 & 591 \\
ZnO NPs & 96 & 380 & 410 & 436 & 580 & 581 \\
\hline
\end{tabular}

${ }^{\mathrm{a}}$ Ref. [48]

of $\mathrm{ZnO}$ NPs obtained under the $633 \mathrm{~nm}$ excitation at room temperature. The phonon peaks observed in bulk $\mathrm{ZnO}$ and our $\mathrm{ZnO}$ NPs cases are summarized in Table 5. A Levenberg-Marquardt nonlinear iteration fitting is performed. The dominating modes in Raman spectrum are $E_{2}($ low $)$ at $96 \mathrm{~cm}^{-1}$ and $E_{2}$ (high) at $436 \mathrm{~cm}^{-1}$ with FWHM $=29 \mathrm{~cm}^{-1}$. The $E_{2}$ (high) modes are attributed to $\mathrm{ZnO}$ non-polar optical phonon, indicating that the sample has a perfect crystal quality [47]. The shift in the position of the $E_{2}$ (high) phonon mode from the bulk may be attributed to the compressive stress in the ZnO NPs. The origin of this red-shift can be ascribed to optical phonon confinement effects [48, 49]. A band displays as a shoulder at about $410 \mathrm{~cm}^{-1}$, that is qualified to the $E_{1}(\mathrm{TO})$ mode. The broad peaks at about 380 and $581 \mathrm{~cm}^{-1}$ are attributed to $A_{1}(\mathrm{TO})$ and $E_{1}(\mathrm{LO})$ modes, respectively, which are associated with oxygen deficiency. Additional Raman modes are observed at $650 \mathrm{~cm}^{-1}$ and $935 \mathrm{~cm}^{-1}$. These peaks originate from acetate groups present in reactant traces used in the synthesis and are ascribed to the OCO symmetric bend and $\mathrm{C}-\mathrm{C}$ symmetric stretch. The peak at
$276 \mathrm{~cm}^{-1}$ is related to the $B_{2} \mathrm{~s}$ order mode with low intensity. The broad feature between 1100 and $1200 \mathrm{~cm}^{-1}$ is assigned to the two phonon modes (2LO), characteristic of II-IV semiconductors [8, 50].

\section{Conclusion}

The zinc oxide nanostructure is successfully synthesized by using sol-gel method with hexagonal wurtzite structure in nanosize range. The synthesized $\mathrm{ZnO}$ NPs obtained exhibit a good crystallinity and small size. Scherrer's formula, W\&H (UDM-USDM) and SSP analysis are helpful to determine the size, the stress and the strain with some approximations. UDM and SSP are highly inter-correlated and confirm the small size of our ZnO NPs. The strain is assumed to be uniform in all crystallographic directions, thus considering the isotropic nature of the crystal. Furthermore, the photoluminescence (PL) characterization of the $\mathrm{ZnO}$ nanostructures exhibits a broad blue emission in the UV range with maximum peak at $490 \mathrm{~nm}$, which is 
attributed to the presence of oxygen anti-site vacancy defect. The size of the ZnO NPs determined by DSL is the about $6 \mathrm{~nm}$. In addition, in Raman spectroscopy, the peak at $436 \mathrm{~cm}^{-1}$ corresponds to $E_{2}$ (high), shifted by $3 \mathrm{~cm}^{-1}$ compared to bulk, that is appropriate to the quantum confinement effect. As a result, the surfactant and the capping agent have an effect on the size, the crystallinity and the luminescence of $\mathrm{ZnO}$ NPs.

Open Access This article is distributed under the terms of the Creative Commons Attribution 4.0 International License (http://creative commons.org/licenses/by/4.0/), which permits unrestricted use, distribution, and reproduction in any medium, provided you give appropriate credit to the original author(s) and the source, provide a link to the Creative Commons license, and indicate if changes were made.

\section{References}

1. Maia, A., Ochoa, M., Portugal, A., Durães, L.: Nanocrystalline $\mathrm{ZnO}$ thin films-influence of sol-gel conditions on the underlying chemistry and film microstructure and transparency. Mater. Today Proc. 2, 49-56 (2015)

2. Gaspara, D., Pereiraa, L., Gehrkeb, K., Gallerb, B., Fortunatoa, E., Martinsa, R.: High mobility hydrogenated zinc oxide thin films. Sol. Energy Mater. Sol. Cells 163, 255-262 (2017)

3. Hariwal, R.V., Malik, H.K., Negi, A., Kandasami, A.: Controlling room temperature ferromagnetism and band gap in $\mathrm{ZnO}$ nanostructured thin films by varying angle of implantation. RSC Adv. 8, 6278-6287 (2018)

4. Chatterjee, A., Shen, C.H., Ganguly, A., Chen, L.C., Hsu, C.W., Hwang, J.Y., Chen, K.H.: Strong room-temperature UV emission of nanocrystalline $\mathrm{ZnO}$ films derived from a polymeric solution. Chem. Phys. Lett. (2004). https://doi.org/10.1016/j.cplett.2004. 05.021

5. Raji, R., Gopchandran, K.G.: ZnO nanostructures with tunable visible luminescence: effects of kinetics of chemical reduction and annealing. J. Sci. Adv. Mater. Dev. 2, 51-58 (2017)

6. Taheri, M., Abdizadeh, H., Golobostanfard, M.R.: Formation of urchin-like $\mathrm{ZnO}$ nanostructures by sol-gel electrophoretic deposition for photocatalytic application. J. Alloys Compd. 725, 291-301 (2017)

7. Siswanto, N., Rochman, T., Akwalia, P.R.: Fabrication and characterization of zinc oxide $(\mathrm{ZnO})$ nanoparticle by sol-gel method. J. Phys. Conf. Ser. (2017). https://doi.org/10.1088/ 17426596/853/1/012041

8. Rajagopalan, P., Singh, V.: Enhancement of $\mathrm{ZnO}$ based flexible nano generators via sol gel technique for sensing and energy harvesting applications. Nanotechnology (2018). https://doi.org/ 10.1088/1361-6528/aaa6bd

9. Dhanashri, P., Vinita, D., Shanabhau, B., Priyanka, P., Prabhakar, S., Madhav, W.: Dimethylamine controlled sol-gel process to grow ZnO nanorods. J Nanostruct. 8, 11-20 (2018)

10. Muchuweni, E., Sathiaraj, T.S., Nyakotyo, H.: Synthesis and characterization of zinc oxide thin films for optoelectronic applications. Heliyon (2017). https://doi.org/10.1016/j.heliyon. 2017.e00285

11. Jońca, J., Ryzhikov, A., Kahn, M.L., Fajerwerg, K., Chaudret, B., Chapelle, A., Menini, P., Fau, P.: Shape-controlled ZnO nanostructures for gas sensing applications. Proc. Eng. 87, 907-910 (2009)
12. Qi, K., Cheng, B., Yu, J., Ho, W.: Review on the improvement of the photocatalytic and antibacterial activities of $\mathrm{ZnO}$. J. Alloys Compd. 15, 792-820 (2017)

13. Koçak, A., Karazu, B.: General evaluations of nanoparticles. ECJSE 5(1), 191-236 (2018)

14. Khan, S.B., Asiri, A.M., Akhtar, K.: Development and prospective applications of nanoscience and nanotechnology. In: Nanomaterials and their Fascinating Attributes (eds) Bentham Science Publishers LTD. Sharjah, UAE, pp. 261 (2015)

15. Yang, W., Wang, L., Lu, X., Zhang, Q.: Enhancing luminescence of $\mathrm{ZnO}$ quantum dots by PEG and oleic acid via a sol-gel method. J. Mater. Sci. Mater Electron. 26, 1113-1118 (2014)

16. Vidyasagar, C.C., Naik, Y.A.: Surfactant (PEG 400) effects on crystallinity of $\mathrm{ZnO}$ nanoparticles. Arab. J. Chem. 9, 507-510 (2016)

17. Salman, M.S., Riaz, A., Iqbal, A., Zulfiqar, S., Sarwar, M.I., Shabbir, S.: Design and fabrication of covalently linked PEGylated nanohybrids of $\mathrm{ZnO}$ quantum dots with preserved and tunable fluorescence. Mater. Des. 131, 156-166 (2017). https:// doi.org/10.1016/j.matdes.2017.06.019

18. Waseda, Y., Matsubara, E., Shinada, K.: X-Ray diffraction crystallography, Introduction, Examples and Solved Problems. Springer, Berlin, Heidelberg (2011)

19. Cullity, B.D.: Element of X-Ray diffraction: Practical Approch. Addison-Wesley Publishing Company Inc., California (1956)

20. Venu Gopal, V.R., Susmita, K.: Effect of temperature on the morphology of $\mathrm{ZnO}$ nanoparticles: a comparative study. Appl. Nanosci. 7, 75-82 (2017)

21. Sahai, A., Goswami, N.: Structural and vibrational properties of $\mathrm{ZnO}$ nanoparticles synthesized by chemical precipitation method. Phys. E 58, 130-137 (2014)

22. Ozutok, F., Demirselcuk, B., Sarica, E., Turkyilmaz, S., Bilgin, V.: Study of ultrasonically sprayed $\mathrm{ZnO}$ films: thermal annealing effect. Acta Phys. Pol. A 121, 53-55 (2012)

23. Prabhu, Y.T., Rao, K.V., Kumar, V.S.S., Kumari, B.S.: X-ray analysis by Williamso-Hall and size-strain plot methods of $\mathrm{ZnO}$ nanoparticles with fuel variation. World J. Nano Sci. Eng. 4, 21-28 (2014)

24. Irfan, H., Racik, K.M., Anand, S.: Microstructural evaluation of CoAl2O4 nanoparticles by Williamson-Hall and size-strain plot methods. J. Asian Ceram. Soc. (2018). https://doi.org/10.1080/ 21870764.2018.1439606

25. Kumar, R., Hymavathi, B.: X-ray peak profile analysis of solidstate sintered alumina doped zincoxide ceramics by WilliamsonHall and size-strain plot methods. JASCER, 260 (2017)

26. Kazeminezhad, I., Saadatmand, I.S., Yousfi, R.: Effect of transition metal elements on the structural and optical properties of $\mathrm{ZnO}$ nanoparticles. Bull. Mater. Sci. 39(3), 719-724 (2015). https://doi.org/10.1007/s12034-016-1206-y

27. Yogamalar, R., Srinivasana, R., Vinub, A., Ariga, A., Bose, A.C.: $\mathrm{X}$-ray peak broadening analysis in $\mathrm{ZnO}$ nanoparticles. Solid State Commun. 149, 1919-1923 (2009)

28. Mote, V.D., Purushotham, Y., Dole, B.N.: Williamson-Hall analysis in estimation of lattice strain in nanometer-sized $\mathrm{ZnO}$ particles. J. Theo. Appl. Phys. 6, 6 (2012)

29. Khorsand Zak, A., Abd Majid, W., Abrishami, M., Yousefi, R.: $\mathrm{X}$-ray analysis of $\mathrm{ZnO}$ nanoparticles by Williamson-Hall and size-strain plot methods. Ceram. Int. 39, 1371-1377 (2013)

30. Sarma, H., Sarma, K.C.: X-ray peak broadening analysis of $\mathrm{ZnO}$ nanoparticles derived by precipitation method. Int. J. Sci. Res. Public. 4, 7 (2014)

31. Yousefi, R.R., Zak, A.K., Jamali-Sheini, F.: The effect of group-I elements on the structural and optical properties of $\mathrm{ZnO}$ nanoparticles. Ceram. Int. 39, 1371-1377 (2013) 
32. Chen, C.Q., Shi, Y., Zhang, Y.S., Zhu, J., Yan, Y.J.: Size dependence of Young's modulus in $\mathrm{ZnO}$ nanowires. PRL 96, 075505 (2006)

33. Nabiyouni, G., Barati, A., Saadat, M.: Surface adsorption of polyethylene glycol and polyvinyl alcohol with variable molecular weights on zinc oxide nanoparticles. Iran. J. Chem. Eng. 8(1), 20-30 (2011)

34. Liufi, S., Xiao, H.: Investigation of PEG adsorption on the surface of zinc oxide nanoparticles. Powder Technol. 145, 20-24 (2004)

35. Patra, M.K., et al.: Synthesis of stable dispersion of $\mathrm{ZnO}$ quantum dots in aqueous medium showing visible emission from bluish green to yellow. J. Luminescence. 129, 320-324 (2009)

36. Tãbãcaru, A., Musat, V., Nicolae, A., Bogdan, A.V., Surdu, V.A.: Vinyltrimethoxysilane-modified zinc oxide quantum dots with tuned optical properties. Appl. Sur. Sci. 359, 766-773 (2015)

37. Huang, W., Bai, D., Li, L., Wei, H., Shi, Z., Cheng, H., Li, Y.: The synthesis of ultrasmall ZnO@PEG nanoparticles and its fluorescence properties. J. Sol-Gel Sci. Technol. 74, 718-725 (2015)

38. Wang, M., et al.: Fabrication and characterization of $\mathrm{ZnO}$ nanoclips by the polyol-mediated process. Nanoscale Res. Lett. 13, 47 (2018)

39. Wood, A., Giersig, M., Hilgendorff, M., Vilas-Campos, A., LizMarzan, L.M., Mulvaney, P.: Influence of solvent on the growth of ZnO nanoparticles. Aust. J. Chem. 56, 1051-1057 (2003)

40. Spanhel, L.: Colloidal $\mathrm{ZnO}$ nanostructures and functional coatings: a survey. J. Sol-Gel Sci. Techn. 39, 7-24 (2006)

41. Spanhel, L., Anderson, M.: Semiconductor clusters in the sol-gel process: quantized aggregation, gelation, and crystal growth in concentrated $\mathrm{ZnO}$ colloids. J. Am. Chem. Soc. 113, 2826-2833 (1991)

42. Hu, Z., Oskam, G., Searson, P.C.: Influence of solvent on the growth of $\mathrm{ZnO}$ nanoparticles. J. Colloid. Interf. Sci. 263, 454 (2003)
43. Taunk, P.B., Das, R., Bisen, D.P., Tamrakar, R.K.: Structural characterization and photoluminescence properties of zinc oxide nanoparticles synthesized by chemical route method. J. Radiation Res. Appl. Sci. 8, 433-438 (2015)

44. Moghaddam, E., Youzbashi, A.A., Kazemzadeh, A., Eshraghi, M.J.: Preparation of surface modified $\mathrm{ZnO}$ quantum dots through an ultrasound assisted sol-gel process. Appl. Surf. Sci. (2015). https://doi.org/10.1016/j.apsusc.2015.03.207

45. Shi, H.Q., Li, W.N., Sun, L.W., Liu, Y., Xiao, H.M., Fu, S.Y.: Synthesis of silane surface modified $\mathrm{ZnO}$ quantum dots with ultrastable, strong and tunable luminescence. Chem. Commun. 47, 11921-11923 (2011)

46. Chand, Z.P., Gaur, A.A., Kumar, A.: Structural and optical properties of $\mathrm{ZnO}$ nanoparticles synthesized at different $\mathrm{pH}$ values. J. Alloys Compd. 539, 174-178 (2012)

47. Zhang, R., Yin, P.G., Wang, N., Guo, L.: Photoluminescence and Raman scattering of $\mathrm{ZnO}$ nanorods. Sol. State Sci. 11, 865-869 (2009)

48. Alim, K.A., Fonoberov, V.A., Shamsa, M., Balandin, A.A.: Micro-Raman investigation of optical phonons in $\mathrm{ZnO}$ nanocrystals. J. Appl. Phys. 971, 24313 (2005)

49. Sendi, R.K., Mahmud, S.: Stress control in ZnO nanoparticlebased discs via high-oxygen thermal annealing at various temperatures. J. Phys. Sci. 24, 1-15 (2013)

50. Calizo, I., Alim, K.A., Fonoberov, V.A., Krishnakumar, S., Shams, M., Balandin, A.A., Kurtz, R.: Micro-Raman spectroscopic characterization of $\mathrm{ZnO}$ quantum dots, nanocrystals and nanowires. Proc. SPIE (2007). https://doi.org/10.1117/12.713648

Publisher's Note Springer Nature remains neutral with regard to jurisdictional claims in published maps and institutional affiliations. 